\title{
斯替宁碱的合成方法研究进展
}

\author{
陈静波陈景超谢彦刘娜娜刘丹丹 张洪涁*
}

(云南大学自然资源药物化学教育部重点实验室 昆明 650091)

\begin{abstract}
摘要 具有 $1 H$-吡咯并 $[1,2-a]$ 氮杂草和多取代全氢吲哚结构的斯替宁碱是百部生物碱的代表性结构, 其多环结构和连 续的七个手性中心对不对称合成化学极具挑战性. 按斯替宁碱合成中使用的关键方法分类, 对斯替宁碱的合成研究进 展进行了综述.
\end{abstract}

关键词 斯替宁; 消旋合成; 不对称合成

\section{Research Progress on the Synthesis of Stenine}

\author{
Chen, Jingbo Chen, Jingchao Xie, Yan \\ Liu, Nana Liu, Dandan Zhang, Hongbin*
}

(Key Laboratory of Medicinal Chemistry for Natural Resources, Ministry of Education, Yunnan University, Kunming 650091)

\begin{abstract}
The alkaloid stenine which has a representative pyrrolo[1,2-a]azepine nucleus and a highly substituted perhydroindole ring system is unique among Stemona alkaloids. The polycyclic system and the seven contiguous stereogenic centers in (一)-stenine present a challenge for asymmetric organic synthesis. Progresses toward the synthesis of stenine are reviewed in terms of the key strategies employed.

Keywords stenine; racemic synthesis; asymmitric synthesis
\end{abstract}

百部是传统中药, 其中所含有的生物碱具药用价 值. 百部生物碱结构的复杂性和多样性引起了合成化学 家的高度重视, 各种类型百部生物碱的合成方法已被相 继报道 ${ }^{[1]}$. 斯替宁碱(Stenine, 亦称百部次碱)为一四环

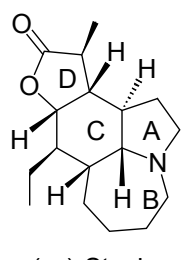

$$
\text { (-)-Stenine }
$$
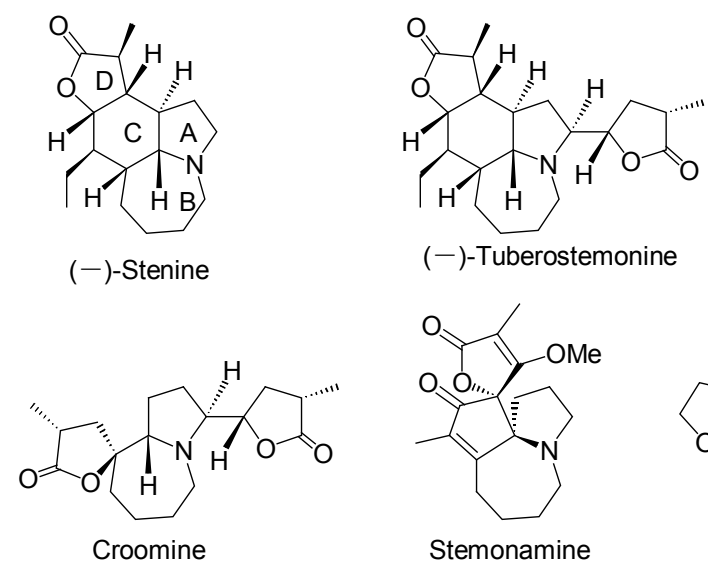

Stemonamine

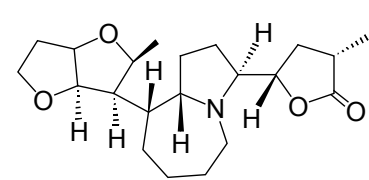

Parvistemonine
生物碱, 分子中有 7 个连续的不对称碳中心, 其 $\mathrm{ABC}$ 环 为核心骨架结构, 在百部生物碱结构中具有代表性, 其 它大多数百部生物碱(图 1)通常有与斯替宁碱相同或是 相似的结构单元.
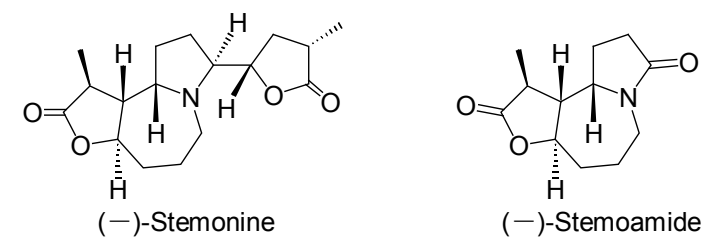

图 1 代表性百部生物碱

Figure 1 Selected stemna alkaloids

\footnotetext{
*E-mail: zhonghb@ynu.edu.cn

Received March 31, 2013; revised May 21, 2013; published online May 24, 2013.

Project supported by the the National Natural Science Foudation of China (Nos. 21062029, 20562013, 20925205).

国家自然科学基金(Nos. 21062029, 20562013 及 20925205)资助项目.
} 
斯替宁碱自 1967 年结构鉴定以来 ${ }^{[2]}$, 其复杂的多环 结构及连续的七个不对称中心激发了合成化学家的浓 厚兴趣. 自从 1990 年 Chen 和 Hart ${ }^{[3]}$ 完成外消旋斯替宁 碱的全合成以来, 斯替宁碱的合成一直方兴未艾 ${ }^{[3 \sim 10]}$. 本文按合成中使用的关键方法分类综述了斯替宁碱的 全合成研究进展, 百部酰胺(Stemoamide)等结构较简单 (三环及三环以下)的百部碱的合成可参见文献[1].

\section{1 斯替宁碱消旋体的合成}

迄今为止, 在外消旋斯替宁碱的全合成中, 全部采 用了以 Diels- Alder 反应为关键步骤的合成方法.

\subsection{Hart 研究组的合成方法}

斯替宁碱(Stenine)于 1990 年由 Hart 研究小组 ${ }^{[3]}$ 首次 合成, 其关键步骤是分子内的 Diels-Alder 反应(Scheme 1), 这一利用 Diels-Alder 反应来构建斯替宁碱六元碳环 (C 环)的策略为后来的研究组广泛采用. 他们利用经一 步酯化反应获得的四烯化合物 $\mathbf{1}$ 在 Lewis 酸 $\left(\mathrm{Me}_{2} \mathrm{AlCl}\right)$ 催化下发生 Diels-Alder 反应, 生成双环化合物 2, 化合 物 2 与肼反应打开内酯环成酰肼, 全甲基化后用乙酰基 保护伯醇羟基得化合物 $\mathbf{3}$, 酰肼化合物 $\mathbf{3}$ 经 Curtius 重排 得氨基甲酸酯化合物 $\mathbf{4}$; 化合物 4 经硼氢化氧化成醇、 醇经甲磺酰化得化合物 $\mathbf{5}$, 然后在甲基锂作用下关环得
到 $\mathrm{AC}$ 双环体系中间体 $\mathbf{6}$, 中间体 $\mathbf{6}$ 经氧化成羒酸化合 物, 与碘作用生成碘代内酯化合物 8 , 再经 DBU 脱碘成 双键、内酯还原和伯醇选择性硅梄保护得烯醇化合物 $\mathbf{9}$, 9 与 $N, N$-二甲基乙酰胺二甲基缩醛发生 EschenmoserClaisen 重排反应的产物经磑代内酯化得碘代物 10, 10 与烯丙基锡试剂发生自由基反应引入烯丙基, 然后在二 异丙基氨基锂(LDA)作用下与碘甲烷反应在酯基的 $\alpha$ 位 引入甲基, 然后经 Swern 氧化、Wittig 反应和红铝选择 性还原得化合物 11, 去除氮原子上保护基后加热内酰 胺化建立七元 $\mathrm{B}$ 环, 烯丙基经氧化断裂形成具有 $\mathrm{ABC}$ 三环体系结构的醛 12,12 的醛基转化为二硫环戊烷、再 与 Lawesson 试剂作用将酰胺转化为硫代酰胺, 最后经 活性镍还原脱硫完成了消旋斯替宁碱 $( \pm)$-Stenine 的第 一次全合成 (Scheme 1). Hart 的合成路线共涉及 28 步反 应，总收率约 $8 \%$, 其通过碘代内酯化引入五元内酯环、 Keck 反应引入烯丙基后再转化为乙基侧链的方法为后 来很多研究组合成百部生物碱时所采用.

\subsection{Jung 研究组 BC 环体系的合成方法}

2000 年, 韩国学者 Jung 等 ${ }^{[6]}$ 采用 Diels-Alder 反应 形成双环酮，继而用 Beckmann 重排的方法构建斯替宁 碱的 $\mathrm{BC}$ 环体系, 再形成 $\mathrm{ABC}$ 三环结构. 但关键步骤分

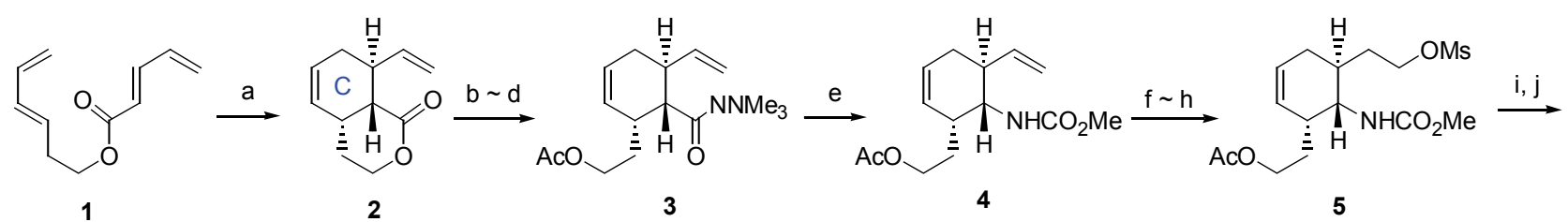<smiles>CC(=O)N1CC[C@H]2CC=C[C@H](CC(=O)O)[C@H]2N1C(C)=O</smiles>

3

4

5
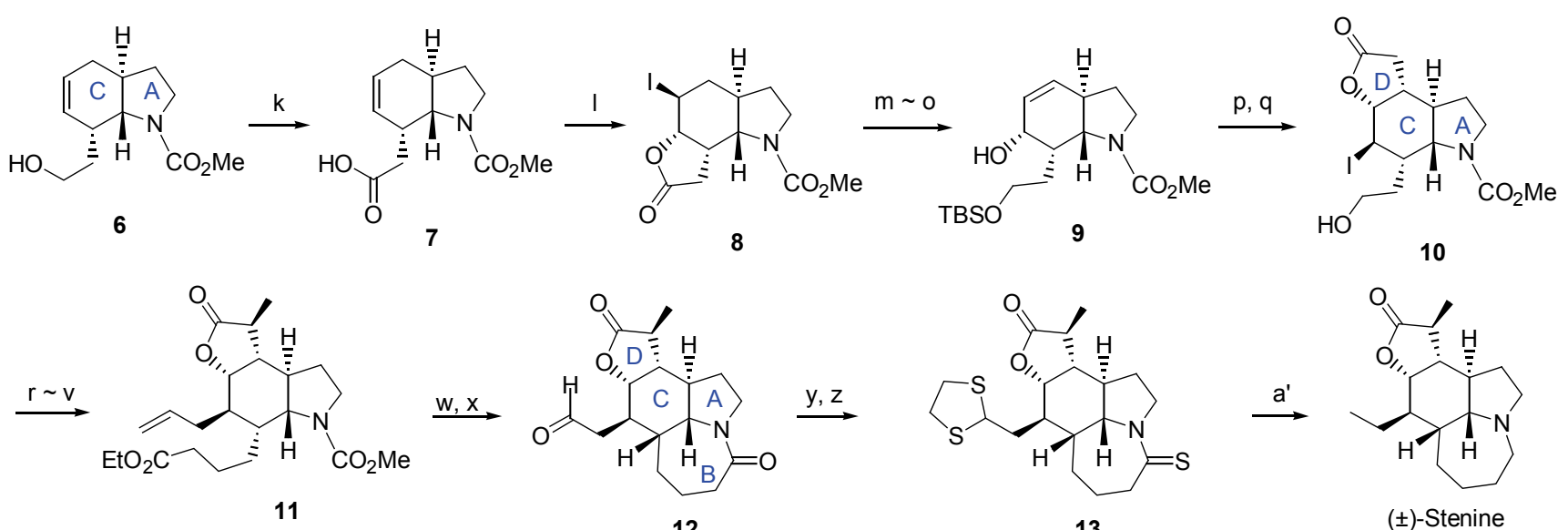

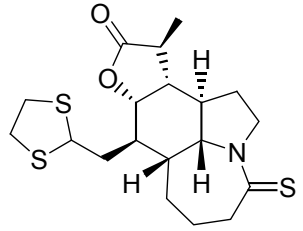

13

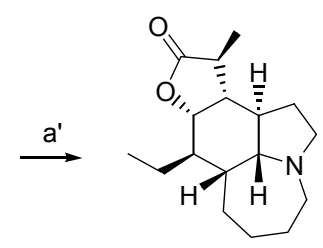

( \pm -Stenine

Reagents and conditions: (a) $\mathrm{Et}_{2} \mathrm{AlCl}$, xylene, reflux (46\% 67\%); (b) $\mathrm{NH}_{2} \mathrm{NH}_{2}$; (c) Mel, $\mathrm{K}_{2} \mathrm{CO}_{3}$; (d) $\mathrm{CH}_{3} \mathrm{COCl}(87 \%, 3$ steps); (e) mesitylene, heat (94\%); (f) 9-BBN; (g) $\mathrm{NaBO}_{3} \cdot 4 \mathrm{H}_{2} \mathrm{O}$; (h) $\mathrm{MsCl}, \mathrm{Et}_{3} \mathrm{~N}$ (95\%, 2 steps); (i) MeLi; (j) $\mathrm{K}_{2} \mathrm{CO}_{3}, \mathrm{MeOH}\left(94 \%, 2\right.$ steps); (k) $\mathrm{CrO}_{3}, \mathrm{H}_{2} \mathrm{SO}_{4}$ (83\%); (l) $\mathrm{I}_{2}$, $\mathrm{NaHCO}_{3}(95 \%)$; (m) DBU; (n) $\mathrm{NaBH}_{4}$; (o) TBDMSCl (95\%, 3 steps); (p) MeC(OMe) $2 \mathrm{NMe}_{2}$, xylenes, reflux (93\%); (q) $\mathrm{I}_{2}, \mathrm{THF}, \mathrm{H}_{2} \mathrm{O}(75 \%) ;(\mathrm{r})$ $n-\mathrm{Bu}_{3} \mathrm{SnCH}_{2} \mathrm{CH}=\mathrm{CH}_{2}$, AIBN (83\%); (s) LDA, THF, Mel (87\%); (t) $\mathrm{Et}{ }_{3} \mathrm{~N}$, DMSO, (COCl) 2 (99\%); (u) $\mathrm{Ph}_{3} \mathrm{P}=\mathrm{CHCO}_{2} \mathrm{Et}, \mathrm{CHCl}_{3}$, reflux (91\%); (v) Red-Al, $\mathrm{CuBr}$ (85\%); (w) Me $\mathrm{Me}_{3} \mathrm{Sil}$, DCM, then mesitylene, heat (86\%); (x) $\mathrm{OsO}_{4}$ (Cat.), $\mathrm{NalO}_{4}(84 \%) ;(\mathrm{y}) \mathrm{HSCH}_{2} \mathrm{CH}_{2} \mathrm{SH}, \mathrm{SiO}_{2}-\mathrm{SO}_{2} \mathrm{Cl}(100 \%) ;(\mathrm{z})$ $\left(p-\mathrm{MeOC}_{6} \mathrm{H}_{4} \mathrm{PS}_{2}\right)_{2}(100 \%)$; (a') W-2 Raney Ni, EtOH (80\%)

\section{Scheme 1}


子内 Diels-Alder 反应收率偏低(62\%), 而较多地使用保 护基亦不利于原子经济性的考量(Scheme 2). 目前尚未 见其将关键中间体 $\mathbf{1 7}$ 转化为 Stenine 的报道.

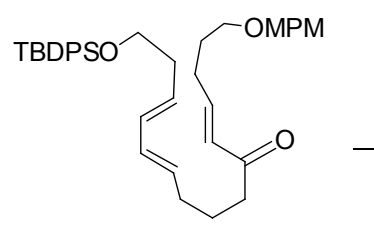

14

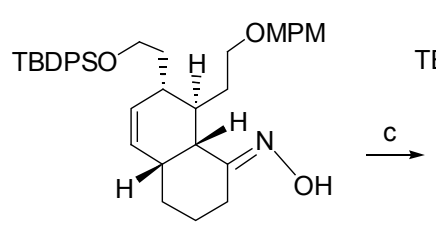

16

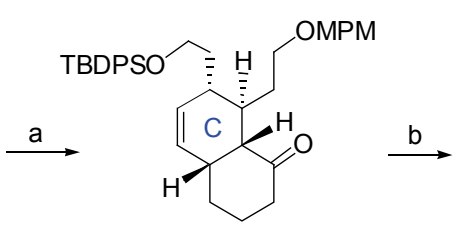

15

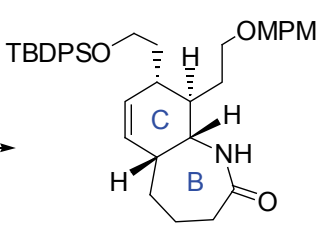

17
Reagents and conditions: (a) $\mathrm{PhCl}$, reflux; (b) $\mathrm{NH}_{2} \mathrm{OH} \cdot \mathrm{HCl}$, pyridine; (c) TsCl, pyridine

\section{Scheme 2}

\subsection{Padwa 研究组的合成方法}

2002 年, 美国埃默里大学 Padwa 小组 ${ }^{[7]}$ 同样利用 [4+2]-环化反应来构建斯替宁的 ABC 三环体系结构化 合物 22, 其环合反应收率为 $85 \%$, 从化合物 $\mathbf{1 8}$ 出发经 18 步反应, 以总收率 $2.1 \%$ 合成了斯替宁碱 $( \pm)$-Stenine (Scheme 3). Padwa 小组利用 $N$-三甲硅基庚内酰胺(18)与 乙二醛二甲硫基单缩醛经 Adol 缩合和乙酰化得化合物
(19)，再与 3- $(E)$-已烯-1,6-二酸单甲酯单酰氯作用得酰 亚胺(20), 20 在 DMTSF [dimethyl(methyl)thiosulfonium tetrafluoroborate]作用下形成的硫正离子引发环化反应 生成二氢呋喃中间体，自发脱除甲硫醇后生成的呋喃化 合物 21 不稳定，室温下很快经 Diels-Alder 反应和重排 反应生成具有 $\mathrm{ABC}$ 三环体系结构的化合物 22, 经还原 脱硫、羰基还原、氢化、脱水等过程生成烯化合物 $\mathbf{2 5}$, 然 后经 Hart 的方法[见 Scheme 1 中 $(\mathrm{q}) \sim(\mathrm{s})$ 及 $(\mathrm{x}) \sim\left(\mathrm{a}^{\prime}\right)$ ]转 化为 $( \pm)$-Stenine.

\subsection{Aubé 研究组的合成方法}

2002 年, 美国堪萨斯大学 Aubé 研究组 ${ }^{[8]}$ 以经 6 步 反应(收率 50\%)合成的三烯酮 26 为中间体，利用分子间 Diels-Alder 分子内 Azido-Schmidt 串联反应高效率地建 立了 $\mathrm{ABC}$ 三环体系化合物 27, 但因有其它两个非对映 异构体产生，收率(43\%)不高(Eq. 1).

化合物 27 经 Birch 还原脱苠基后得到醇、醇氧化为 羧酸后经碘代内酯化反应生成具有 $\mathrm{ABCD}$ 四环体系结 构的中间体 30, 然后用 Hart 的方法 [见 Scheme 1 中 $(\mathrm{x}) \sim$ $\left(a^{\prime}\right)$ ]又经四步反应得到 $( \pm)$-Stenine, 整个合成路线共 16 步反应, 是一个比较高效率的合成方法(Scheme 4), 总 收率为 $7.7 \%$.
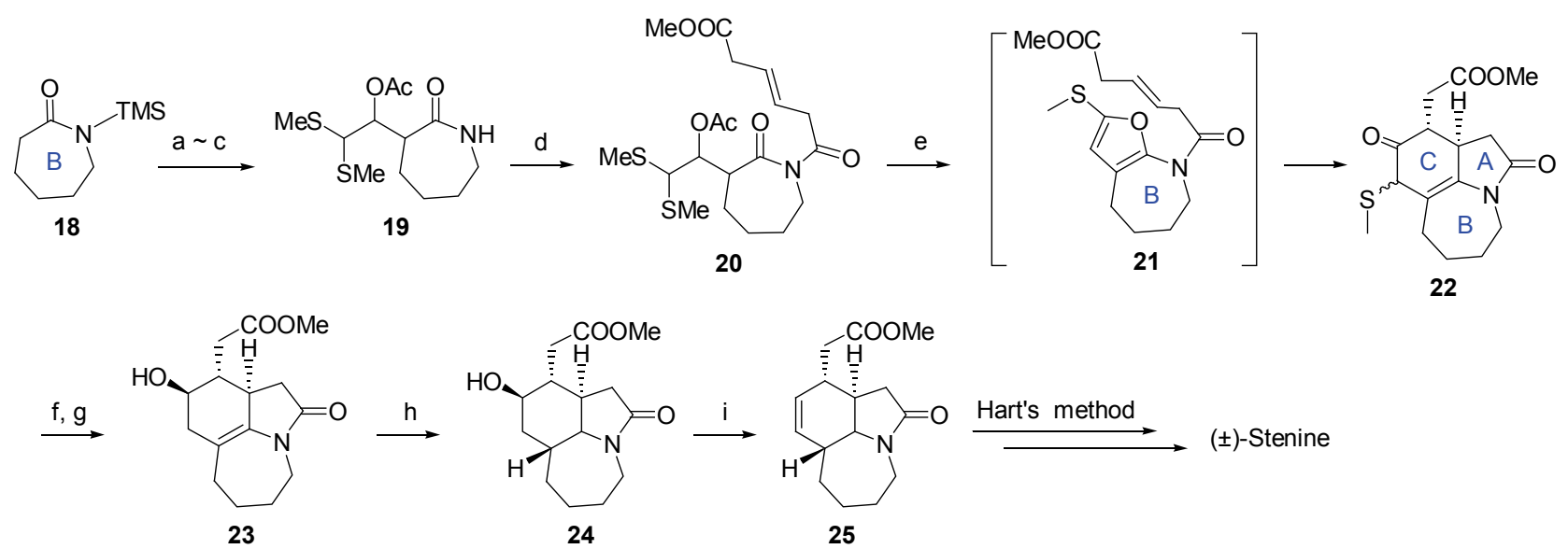

Reagents and conditions: (a) LDA; (b) (MeS) $)_{2} \mathrm{CHCHO;} \mathrm{(c)} \mathrm{Ac}_{2} \mathrm{O}$ (80\%, 3 steps); (d) (E)-methyl 5-(chlorocarbonyl)pent-3-enoate (85\%, 3 steps); (e) DMTSF, $\mathrm{Et}_{3} \mathrm{~N}$ (80\%); (f) R-Ni, EtOH; (g) $\mathrm{NaBH}_{4}, \mathrm{CeCl}_{3}\left(71 \%, 2\right.$ steps); (h) Crabtree's catalyst, $\mathrm{H}_{2}$; (i) $\mathrm{MsCl}_{1} \mathrm{Et}_{3} \mathrm{~N}, \mathrm{DBU}(64 \%, 2$ steps)

Scheme 3

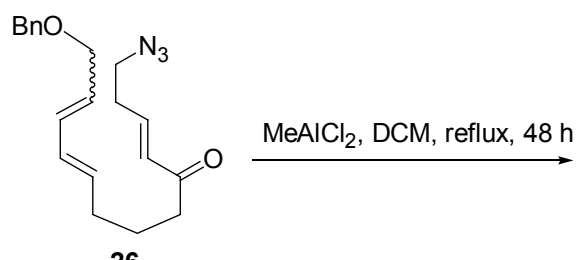

26

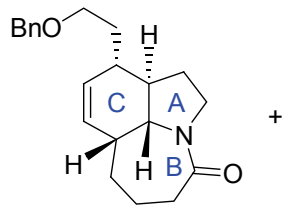

$27(43 \%)$

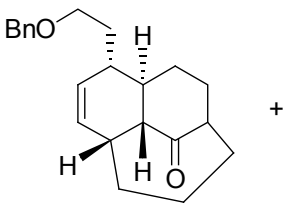

$28(24 \%)$

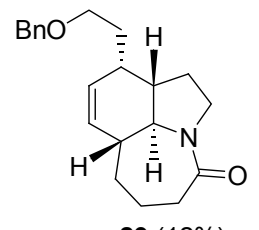

$29(12 \%)$ 


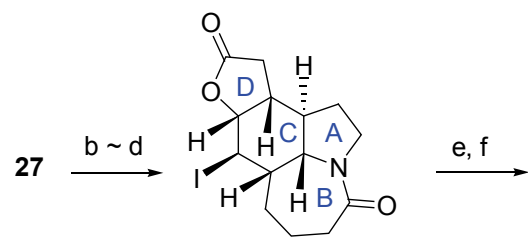

30

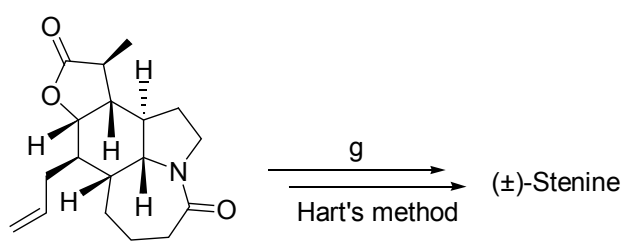

31

Reagents and conditions: (b) $\mathrm{Na}, \mathrm{NH}_{3}$ (liq.); (c) $\mathrm{CrO}_{3}, \mathrm{H}_{2} \mathrm{SO}_{4}$; (d) I $\mathrm{I}_{2} \mathrm{NaHCO}_{3}(80 \%, 3$ steps); (e) allyltributyltin, AlBN, benzene; (f) LHMDS, Mel ( $72 \%, 2$ steps), (g) Hart's method (63\%, 4 steps)

\section{Scheme 4}

2005 年和 2008 年该研究组 ${ }^{[9]}$ 对合成路线进行了改 进, 在 Diels-Alder 反应的原料一二烯体中预设了一个 乙基, 同样经分子间 Diels-Alder 反应和分子内 AzidoSchmidt 串联反应高效构建了带有乙基侧链的 $\mathrm{ABC}$ 三环 体系化合物 36; 在后续的转化中省去了 Hart 方法中烯 丙基化和从烯丙基向乙基转化的 4 步反应，从商品化原 料出发共 9 步反应完成了外消旋斯替宁碱的合成 (Scheme 4), 总收率 $14 \%$, 是目前效率最高的 $( \pm$ )Stenine 的合成方法; 此外, 36 的非对映异构体 $\mathbf{3 5}$ 经 10 步反应首次完成了新斯替宁碱(Neostenine)的全合成 (Scheme 5).<smiles>CCCC(=O)CP(=O)(OC)OC</smiles>

32<smiles>CC[C@H]1C(=O)C[C@@H]2CCN3C(=O)CCC[C@@H]1[C@]23C</smiles>
$35(18 \%)$

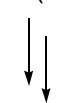

( \pm )-Neostenine<smiles>N#CCC=O</smiles>

33

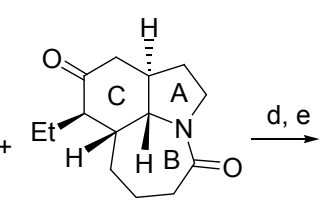

$36(53 \%)$

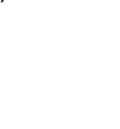

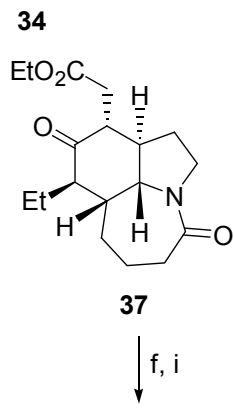

$( \pm)$-Stenine
Reagents and conditions: (a) NaH; (b) TMSOTf (43\%, 2 steps); (c) $\mathrm{SnCl}_{4}$, cyclohexenone; (d) LiHMDS; (e) $\mathrm{BrCH}_{2} \mathrm{CO}_{2} \mathrm{Et}$ (73\%, 2 steps); (f) $\mathrm{NaBH}_{4}$; (g) LiHMDS, Mel; (h) Lawesson's reagent; (i) Raney Ni (42\%, 4 steps).

\section{Scheme 5}

\subsection{Shea 研究组 BCD 三环体系的合成方法}

2007 年, Shea 研究组 ${ }^{[10]}$ 报道了一种杂原子参与的分 子内 Diels-Alder 反应环化产物经还原 $\mathrm{N}$ - $\mathrm{O}$ 键裂解首先 形成 $\mathrm{BC}$ 二环体系化合物 $\mathbf{4 0}$, 然后经 5 步反应构建了斯 替宁碱的 BCD 三环体系(Scheme 6)的方法.

\section{2 斯替宁碱的不对称合成}

斯替宁碱的不对称合成方法从策略来看可分为三

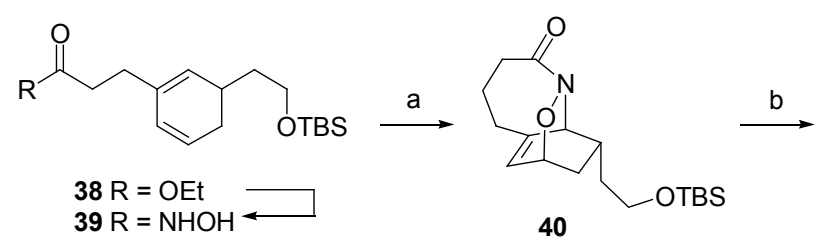

$\mathrm{HO}$,

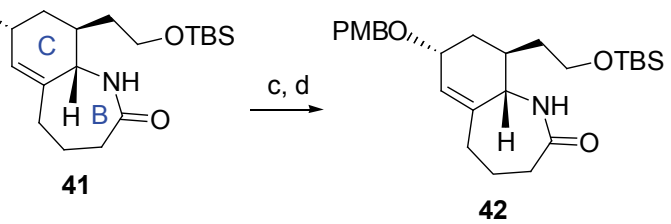

PMBO,

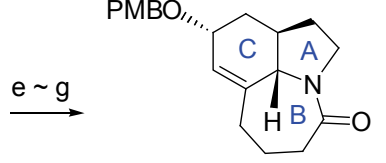

43

Reagents and conditions: (a) $\mathrm{Bu}_{4} \mathrm{NIO}_{4}(50 \%, d r=6: 1)$; (b) $\mathrm{Na} / \mathrm{Hg}$, $\mathrm{Na}_{2} \mathrm{HPO}_{4}$ (51\%); (c) PMB-OC(=NH)Cl ; (d) $\mathrm{Ph}_{3} \mathrm{CBH}_{4}(51 \%, 2$ steps); (e) HF, Py (73\%, 2 steps); (f) $\mathrm{TSCl}, \mathrm{Et}_{3} \mathrm{~N}$; (g) $\mathrm{NaH}(67 \%, 3$ steps).

\section{Scheme 6}

类: 一类是 Wipf 等以天然手性源酪氨酸或其衍生物为 原料，经氧化偶联形成 $\mathrm{AC}$ 二环体系，然后再构建 $\mathrm{B}$ 环 和 D 环; 另一类是多数学者所采用的方法，即通过手性 辅助基团的立体控制作用，经不对称 Diels-Alder 反应等 首先构建合适的双环或三环体系，然后经官能团转化、 环化等反应合成斯替宁碱; 到我们从事斯替宁碱的合成 为止，不对称合成均采用了手性原料或手性辅基的方法 进行. 第三类即为本研究组采用不对称催化反应和串联 环化反应为关键步骤的合成方法，具有立体控制好、合 成效率高的特点.

\subsection{Wipf 研究组利用手性原料的合成方法}

1995 年, 美国匹兹堡大学 Wipf 小组 ${ }^{[4]}$ 从 $N-\mathrm{Cbz}-L-$ Tyrosin (44)出发, 经氧化偶联和分子内 Michael 加成串 联反应立体选择性地合成了八氢吲哚衍生物 $\mathbf{4 5}$, 构筑 了斯替宁碱的 AC 二环体系(Scheme 7). 本方法有效地 利用了天然手性源，并创新性地利用氧化去芳化反应获 得重要的中间体用于百部生物碱的合成. 化合物 45 经 苯甲酸酯化、羰基还原和钯催化还原脱除烯丙位酯基得 
烯丙醇化合物 46.

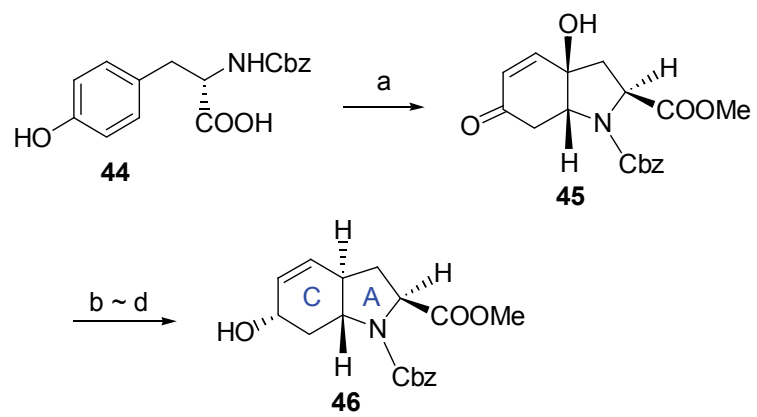

Reagents and conditions: (a) $\mathrm{Phl}(\mathrm{OAc})_{2}, \mathrm{MeOH}, \mathrm{NaHCO}_{3}, 23{ }^{\circ} \mathrm{C}$ (54\%); (b) $\mathrm{Bz}_{2} \mathrm{O}, \mathrm{DCM}, \mathrm{Py}, \mathrm{DMAP}$, reflux (51\%); (c) $\mathrm{NaBH}_{4}$, $\mathrm{CeCl}_{3} \cdot 7 \mathrm{H}_{2} \mathrm{O}, \mathrm{MeOH}$, THF, r.t.; (d) $\mathrm{Pd}_{2}(\mathrm{dba})_{3} \cdot \mathrm{CHCl}_{3}$, THF, $n-\mathrm{Bu}_{3} \mathrm{P}$, $\mathrm{HCO}_{2} \mathrm{H}, \mathrm{Et}_{3} \mathrm{~N}, 60{ }^{\circ} \mathrm{C}(55 \%, 2$ steps $)$

\section{Scheme 7}

化合物 46 氧化成酮后在 $\alpha$ 位进行烷基化, 再次还原 酮羰基成醇后按 Hart 的方法与 $N, N$-二甲基乙酰胺二甲 基缩醛经 Eschenmoser-Claisen 重排反应生成酰胺化合 物 47 (Scheme 8). 酰胺 $\mathbf{4 7}$ 经选择性氧化切断末端双键 成醛、还原成醇后再转变成硅醚 $\mathbf{4 8}$; 酯基水解为羧酸后 再转化为烷氧磷酸的混䣶, 经自由基还原脱除酯得化合 物 49; 化合物 49 经 Hart 的方法 [ 见 Scheme 1 中(q)] 进行
碘代内酯化建立 ACD 三环体系，再经 Keck 烯丙基化方 法立体选择性引入烯丙基得中间体 50; 然后经 8 步反应 转化为(一)-Stenine. 这是从手性原料出发第一次对斯替 宁碱的不对称合成 ${ }^{[4]}$, 共计 26 步反应，最后以 $1.1 \%$ 的总 收率合成了(一)-Stenine (Scheme 8).

\subsection{Morimot 研究组利用手性辅助基团的合成方法}

1996 年, 日本 Morimoto 研究组 ${ }^{[5]}$ 也完成了斯替宁 碱的不对称全合成，仍然采用分子内 Diels-Alder 反应的 合成策略，并在该步反应中引入手性辅基，建立了 $\mathrm{B}$ 环 及 4 个不对称中心. 经 4 步反应获得的三烯化合物 52 在 Lewis 酸 $\left(\mathrm{Me}_{2} \mathrm{AlCl}\right)$ 催化下发生 Diels-Alder 反应，形成 的双环产物非对映异构体比率为 $10 ： 1$, 并具有完全的 内型选择性, 经水解 1,3-二噻烷、还原去除手性辅助基 团、氧化醛成羧酸化合物 53, 53 经 Shioiri 改良的 Crutius 方法将羧基转变为氨基甲酸酯化合物, 继而转化为烯醇 硅醚化合物 54 后，经间氯过氧苯甲酸和亚氯酸钠两次 氧化形成羧酸衍生物, 再经碘代内酯化反应立体选择性 地建立了 $\mathrm{ACD}$ 三环体系化合物 $\mathbf{5 5} ; \mathbf{5 5}$ 的 $N, O$-半缩醛与 原甲酸三乙酯反应，经甲基化转化为 $\mathrm{N}, \mathrm{O}$-缩醛，然后经 Hart 的方法立体选择性引入烯丙基和甲基得化合物 $\mathbf{5 6}$ (Scheme 9).

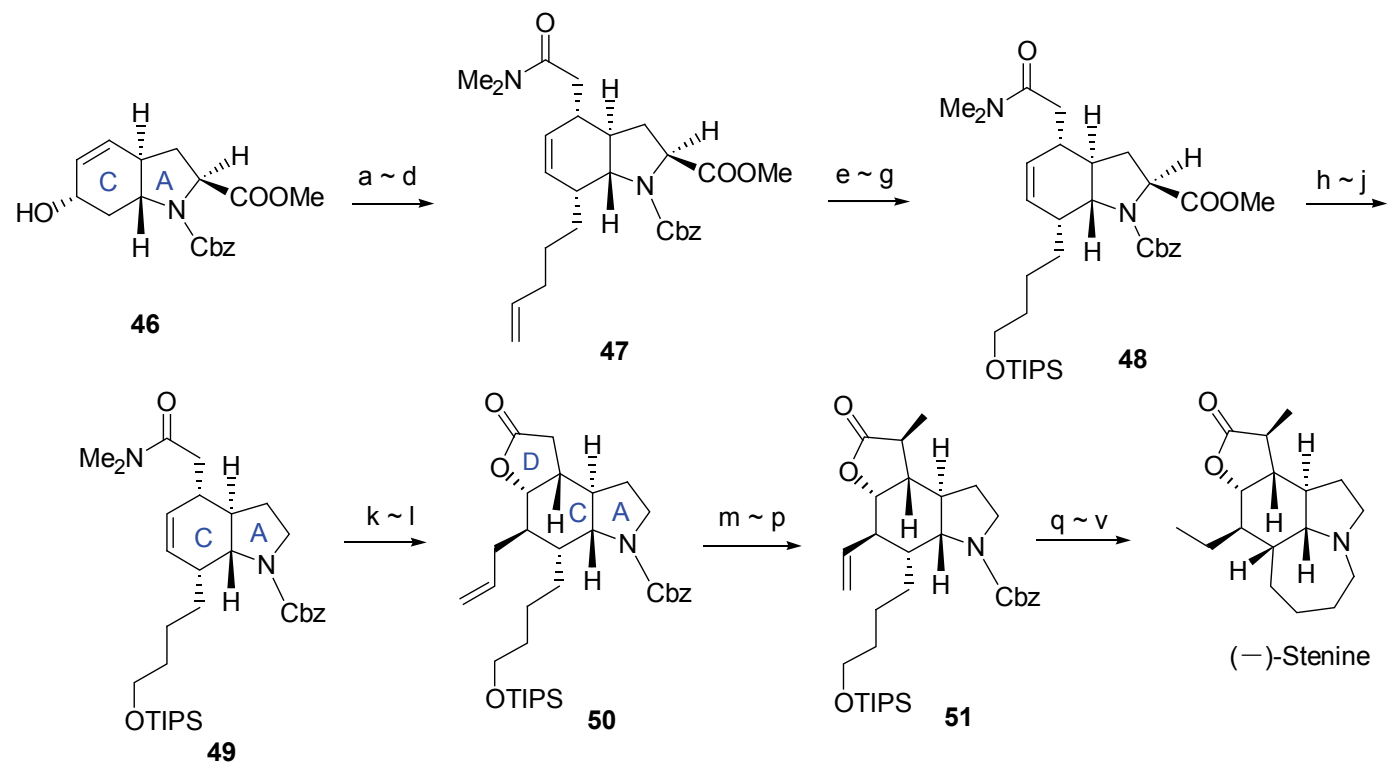

Reagents and conditions: (a) TPAP (cat.), NMO, DCM, MS, $0{ }^{\circ} \mathrm{C}$ to r.t.; (b) KHMDS, toluenen, $-80{ }^{\circ} \mathrm{C}$; then $\mathrm{CH} \mathrm{H}_{2}=\mathrm{CH}\left(\mathrm{CH}_{2}\right)_{3} \mathrm{OTf}$, THF, -60 ${ }^{\circ} \mathrm{C}$; (c) $\mathrm{NaBH}_{4}, \mathrm{CeCl}_{3} \cdot 7 \mathrm{H}_{2} \mathrm{O}$, THF, $\mathrm{MeOH}, 40{ }^{\circ} \mathrm{C}$; (d) $\mathrm{MeC}(\mathrm{OMe}){ }_{2} \mathrm{NMe}_{2}$, xylenes, reflux ( $36 \%, 4$ steps); (e) AD-mix- $\beta, t-\mathrm{BuOH}, \mathrm{H}_{2} \mathrm{O}, 5{ }^{\circ} \mathrm{C}$; then $t$ - $\mathrm{BuOH}, \mathrm{H}_{2} \mathrm{O}, \mathrm{NaIO}_{4}$, r.t.; (f) $\mathrm{NaBH}_{4}, \mathrm{CeCl}_{3} \cdot 7 \mathrm{H}_{2} \mathrm{O}, \mathrm{MeOH}, \mathrm{THF}, 40{ }^{\circ} \mathrm{C}$; (g) TIPSCl, imidazole, DMAP (cat.), DCM, r.t. (76\%, 3 steps); (h) LiOH,

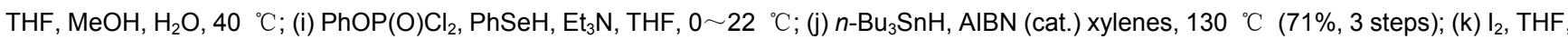
pH 5.5, $21{ }^{\circ} \mathrm{C}$; (l) $\mathrm{CH}_{2} \mathrm{CH}=\mathrm{CH}_{2} \mathrm{SnBu}_{3}$, AIBN (cat.), $80{ }^{\circ} \mathrm{C}$ (77\%, 2 steps); (m) LDA, THF, HMPA, Mel, $-78{ }^{\circ} \mathrm{C}$; (n) OsO (cat.), NalO $_{4}, \mathrm{THF}$, $\mathrm{H}_{2} \mathrm{O}, t$-BuOH, $0 \sim 21{ }^{\circ} \mathrm{C}$; (o) $\mathrm{NaBH}_{4}$, THF, MeOH, $-40{ }^{\circ} \mathrm{C}$; (p) o- $\left(\mathrm{NO}_{2}\right) \mathrm{C}_{6} \mathrm{H}_{4} \mathrm{SeCN}, \mathrm{Bu}_{3} \mathrm{P}, \mathrm{THF}, 0{ }^{\circ} \mathrm{C}$; then $\mathrm{H}_{2} \mathrm{O}_{2}, \mathrm{THF}, 21{ }^{\circ} \mathrm{C}(48 \%, 4 \mathrm{steps})$; (q) $\mathrm{HF}, \mathrm{CH}_{3} \mathrm{CN}, 0{ }^{\circ} \mathrm{C}$; (r) Dess-Martin periodinane, $\mathrm{CH}_{2} \mathrm{Cl}_{2}, 21{ }^{\circ} \mathrm{C}$, then THF, 2-methylbut-2-ene, $\mathrm{NaClO}_{2}$, aq. $\mathrm{Na}_{2} \mathrm{HPO}_{4}, 0{ }^{\circ} \mathrm{C}$; $(\mathrm{s}) \mathrm{H}_{2}$, $\mathrm{Pd}(\mathrm{OH})_{2} / \mathrm{C}, \mathrm{MeOH}, 21{ }^{\circ} \mathrm{C}$; (t) $\mathrm{C}_{6} \mathrm{~F}_{5} \mathrm{P}(\mathrm{O}) \mathrm{Ph}_{2}, \mathrm{CH}_{2} \mathrm{Cl}_{2}, 21{ }^{\circ} \mathrm{C}$ (71\%, 4 steps); (u) (p-MeOC $\left.{ }_{6} \mathrm{H}_{4} \mathrm{PS}_{2}\right)_{2}, \mathrm{CH}_{2} \mathrm{Cl}_{2}, 21{ }^{\circ} \mathrm{C}$ (93\%); (v) Raney Ni, EtOH, $21{ }^{\circ} \mathrm{C}(78 \%)$

\section{Scheme 8}



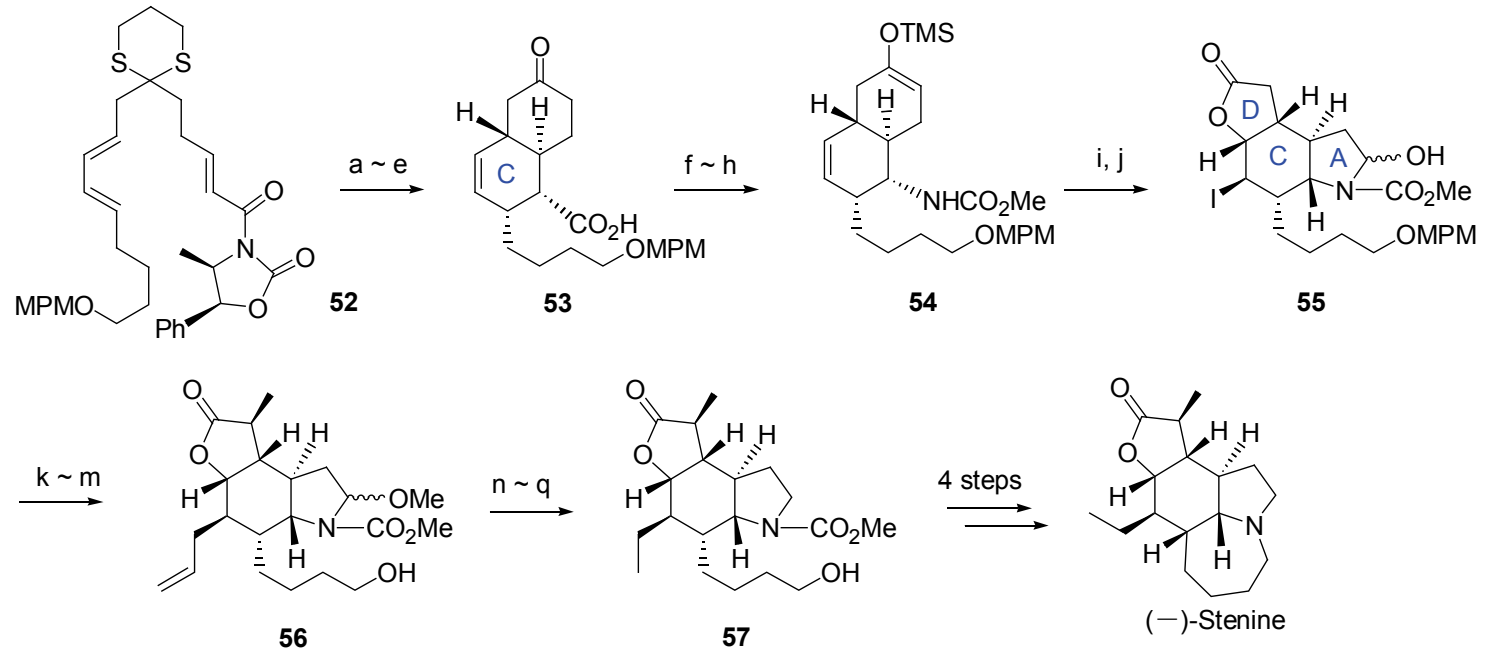

Reagents and conditions: (a) $\mathrm{Me}_{2} \mathrm{AlCl}, \mathrm{DCM},-20{ }^{\circ} \mathrm{C}$ (85\%); (b) $\mathrm{AgNO}_{3}, \mathrm{NCS}, \mathrm{CH}_{3} \mathrm{CN} / \mathrm{H}_{2} \mathrm{O}(80 \%)$; (c) LiSEt, $\mathrm{THF}, 0{ }^{\circ} \mathrm{C}(91 \%) ;(\mathrm{d}) \mathrm{Et} \mathrm{SiH}_{3}$ $10 \% \mathrm{Pd} / \mathrm{C}$, acetone, $0{ }^{\circ} \mathrm{C}$ (100\%); (e) $\mathrm{NaClO}_{2}, \mathrm{NaH}_{2} \mathrm{PO}_{4}$, 2-methyl-2-butene, $t-\mathrm{BuOH} / \mathrm{H}_{2} \mathrm{O}, 0{ }^{\circ} \mathrm{C}$ (100\%); (f) DPPA, NEt 3 , DMF, $60{ }^{\circ} \mathrm{C}, 30 \mathrm{~min}$; (g) 10 equiv. $\mathrm{MeOH}, 0.2$ equiv. $\mathrm{CuCl}$, r.t., $40 \mathrm{~h}$ (82\%, 2 steps); (h) TMSCl, $\mathrm{Nal}, \mathrm{NEt}_{3}$; (i) $m$-CPBA, hexane/DCM $(V: V=2: 1)$, $-15{ }^{\circ} \mathrm{C}$ to r.t., $2 \mathrm{~h}$; (j) $\mathrm{H}_{5} \mathrm{IO}_{6}$, THF/ $\mathrm{H}_{2} \mathrm{O}$, r.t., 2 h, then $\mathrm{I}_{2}, \mathrm{NaHCO}_{3}$, r.t., $40 \mathrm{~h}$ (50\%, 3 steps); (k) 0.05 equiv. $\mathrm{CH}(\mathrm{OMe})_{3}, \mathrm{MeOH}$, DCM, r.t., 1 h; (I) 3 equiv. allyltributyltin, 0.15 equiv. AlBN, toluene, $80{ }^{\circ} \mathrm{C}, 4 \mathrm{~h}$; (m) Li, HMPA, THF, $-78{ }^{\circ} \mathrm{C}, 30 \mathrm{~min}, \mathrm{Mel},-78{ }^{\circ} \mathrm{C}, 1.5 \mathrm{~h}(53 \%, 3 \mathrm{steps})$; (n) $5 \mathrm{equiv}$. Et $3 \mathrm{SiH}$, 2.2 equiv. $\mathrm{BF}_{3} \cdot \mathrm{Et}_{2} \mathrm{O}, \mathrm{CH}_{3} \mathrm{CN}, 0^{\circ} \mathrm{C}, 50 \mathrm{~min}$; (o) Cat., $\mathrm{OsO}_{4}, \mathrm{NalO}_{4}, \mathrm{THF} / \mathrm{H}_{2} \mathrm{O}(V: V=2: 1)$, r.t., $1 \mathrm{~h}$; (p) 1,2-ethanedithiol, $\mathrm{BF}_{3}{ }^{\circ} \mathrm{Et}_{2} \mathrm{O}, \mathrm{DCM},-15$ ${ }^{\circ} \mathrm{C}, 45 \mathrm{~min}$; (q) Raney Ni (W-2) EtOH, reflux, 2 h (42\%, 4 steps).

\section{Scheme 9}

中间体 $\mathbf{5 6}$ 结构中的 $N, O$-缩醛经三乙基硅烷还原去 除甲氧基，烯丙基经 Lemieux-Johnson 氧化、二硫五环 生成和还原脱硫反应转化为乙基得化合物 $\mathbf{5 7}$, 然后经 4 步反应转化为(一)-Stenine. Morimoto 小组共用了 24 步 反应，最后以 $1.8 \%$ 的总收率合成了(一)-Stenine (Scheme 9).

\subsection{Fujioka 研究组利用手性辅助基团的合成方法}

2012 年, 日本大阪大学 Fujioka 小组以卤代醚化等 反应制备的带有手性辅助基团的环已烯酮化合物 $\mathbf{5 8}$ 为 原料, 通过环状手性辅助基团的空间控制作用, 立体选 择性地在羰基 $\beta$-位引入烯丙基得到化合物 59, 再经 Michael 加成反应在羰基 $\beta$-位引入 4-戊烯基构建了五取 代环己酮化合物 60, DDQ 作用下缩醛水解得醛化合物 61; 化合物 61 与对甲氧基芐胺经还原胺化反应所得仲 胺与邻硝基苯磺酰氯作用得化合物 62, 乙腈中与硝酸 铈铵和水作用脱去对甲氧基芐基得化合物 63; 化合物 63 经分子内 Mitsunobu 反应构建了具有多取代全氢吲哚 结构的化合物 64, 强碱条件下与碘乙烷作用在位阻小 的羰基 $\alpha$-位引入乙基得化合物 $\mathbf{6 5}$, 然后经臭氧氧化、还 原分解成醛、PDC 氧化成酸、甲酯化反应得双酯化合物 66; 化合物 66 先与碳酸铯和苯硫酚作用脱去氮原子上 的邻硝基苯磺酰基, 再在甲苯中加热经酰胺环化反应得 三环化合物 67, 然后经还原内酯化得到 13-去甲基-5-
氧一斯替宁 $(68$, Scheme 10). 化合物 68 可参照文献 $[9,12]$ 方法转化为 $(-)$-Stenine.

\section{4 张洪涁研究组利用手性催化的合成方法}

2012 年, 本研究组 ${ }^{[12]}$ 利用手性催化和串联环化反 应为关键方法完成了 $(-)$-Stenine 的不对称全合成 (Scheme 11). 3-丁烯-1-醛二乙基缩醛(69)经臭氧化后还 原得 3,3-二乙氧基缩醛(70), 70 与硝基甲烷在碱性条件 下经 Henry 反应生成硝基醇化合物, 不经纯化的粗产物 在四氢呋喃中与三乙胺和三氟乙酸䣶作用脱水生成硝 基烯化合物 71 (Scheme 11). 参考文献[6]方法，环戊烯 (72)经臭氧氧化后控制还原分解得 4-甲酰基丁酸甲酯 (73).

麦氏酸(Meldrum's acid)与丁酰氯和三乙胺作用生 成缩酮化合物 75, 然后在碱性条件下与氯亚磷酸二乙 酯作用, 再经过氧化氢氧化得到磷酸酯化合物 76, 化合 物 76 与 73 经 Horner-Wadsworth-Emmons 反应生成烯化 合物 77, 其反式与顺式异构比例为 $4: 1$, 化合物 77 在 四氢呋喃中与由甲醇和氢化钠新鲜制备的甲醇钠作用 转化为不饱和酮酸酯 $\mathbf{7 8}$, 反式与顺式异构体经硅胶柱 层析很容易分离, 需要指出的是此结构预设了 ( - ) Stenine 全合成中 B环上的侧链乙基, 为高效合成打下了 良好的基础(Scheme 12). 


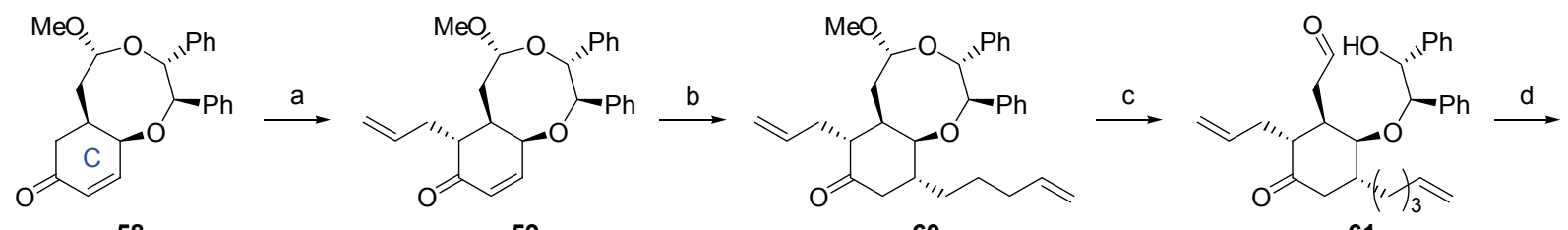

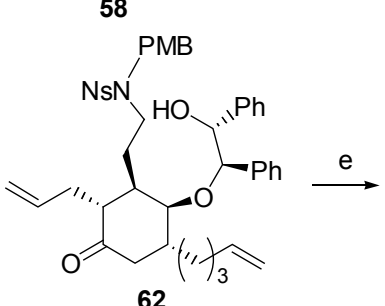

59

60

61

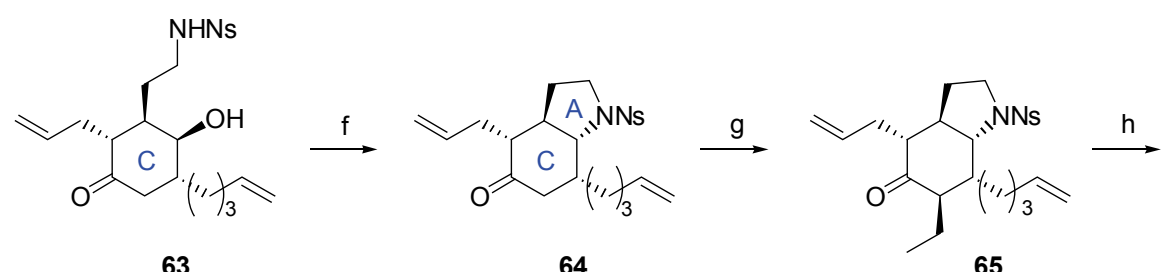

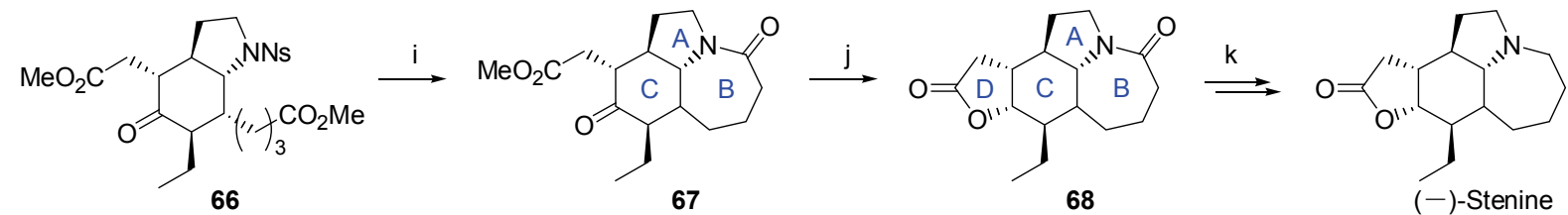

Reagents and conditions: (a) LHMDS, allyl iodide, HMPA, THF, $-78{ }^{\circ} \mathrm{C}, 3 \mathrm{~h}(92 \%)$; (b) 4-pentenyl magnesium bromide, Cul, THF, $-78{ }^{\circ} \mathrm{C}, 3$ h (90\%); (c) $\mathrm{DDQ}, \mathrm{CH}_{3} \mathrm{CN} / \mathrm{H}_{2} \mathrm{O}(V: V=10: 1)$, r.t., $4 \mathrm{~h}(84 \%)$; (d) i) 4-methoxybenzylamine, $\mathrm{NaBH}(\mathrm{OAc})_{3}, 1,2-\mathrm{DCE}$, r.t., 1.5 h; ii) 2-nitrobenzenesulfonyl chloride, $\mathrm{Et}_{3} \mathrm{~N}, \mathrm{CH}_{2} \mathrm{Cl}_{2}$, r.t., $30 \mathrm{~min}$ (73\%, 2 steps); (e) $\mathrm{CAN}, \mathrm{CH}_{3} \mathrm{CN} / \mathrm{H}_{2} \mathrm{O}(\mathrm{V}: \mathrm{V}=2$ : 1), r.t., 5 h (62\%); (f) DIAD, $\mathrm{PPh}$, 1,4-dioxane, r.t., $15 \mathrm{~min}(55 \%)$; (g) LHMDS, ethyl iodide, HMPA, THF, $-78{ }^{\circ} \mathrm{C}$ to r.t., $24 \mathrm{~h}(57 \%, 80 \%)$; (h) i) $\mathrm{O}_{3}, \mathrm{CH}_{2} \mathrm{Cl}_{2},-78{ }^{\circ} \mathrm{C}, 15 \mathrm{~min}$, then $\mathrm{PPh}_{3}$, r.t., $1 \mathrm{~h}(81 \%)$; ii) $\mathrm{PDC}$, DMF, r.t., 24 h; iii) $\mathrm{TMSCHN}_{2}$, benzene/MeOH ( $\left.V: V=4: 1\right)$, r.t., $30 \mathrm{~min}(70 \%, 2 \mathrm{steps})$; (i) i) $\mathrm{CsCO}$, $\mathrm{PhSH}, \mathrm{CH}_{3} \mathrm{CN}$, r.t., $12 \mathrm{~h}$; ii) toluene, reflux, $24 \mathrm{~h}$ (95\%, 2 steps); (j) $\mathrm{NaBH}_{4}, \mathrm{MeOH}, 0{ }^{\circ} \mathrm{C}, 1.5 \mathrm{~h}(64 \%)$; (k) Ref. [9, 12]

\section{Scheme 10}<smiles>C=CCC(OCC)OCC</smiles>

69<smiles>C1=CCCC1</smiles>

72
70<smiles>C1=CCCC1</smiles><smiles>COC(=O)CCCC=O</smiles>

73

Reagents and conditions: (a) $\mathrm{O}_{3}, \mathrm{DCM}$, then $\mathrm{Me}_{2} \mathrm{~S}$; (b) $\mathrm{MeNO}_{2}$, $\mathrm{EtOH}, \mathrm{NaOH}$ (aq.), the crude product was then treated with $\mathrm{Et}_{3} \mathrm{~N}$ and TFAA in THF, $0{ }^{\circ} \mathrm{C}$ (81\%, 2 steps); (c) Ref. [13] (95\%)

\section{Scheme 11}<smiles></smiles>

74<smiles>CCC(=CCCCC(=O)OC)C1=CC(=O)OC(C)(C)O1</smiles>

77: $E / Z=4: 1$<smiles>CCCC1C=C(O[13CH3])OC(C)(C)O1</smiles>

76<smiles>CC/C(=C\CCCC(=O)OC)C(=O)CC(=O)OC</smiles>

78

Reagents and conditions: (a) Butyryl chloride, $\mathrm{Et}_{3} \mathrm{~N}$, toluene (93\%); (b) LiHMDS, CIP(OEt) 2 , then $30 \% \mathrm{H}_{2} \mathrm{O}_{2}(86 \%)$; (c) LDA, THF, -78 ${ }^{\circ} \mathrm{C}$, HMPA, then 62 (85\%); (d) MeOH, NaH, THF (80\%)

\section{Scheme 12}

反式不饱和酮酸酯 78 与硝基烯化合物 71 在手性镍 催化剂 79 作用下发生第一次 Michael 加成反应，然后在 碱性硅胶和超声作用下发生第二次 Michael 加成反应, 经连续的一瓶反应生成烯醇式五取代环己酮化合物 $\mathbf{8 0}$, 对映选择性和非对映选择性良好，其环己烷上取代基的 构象与(一)-Stenine 不对称合成的要求相一致(Eq. 2).

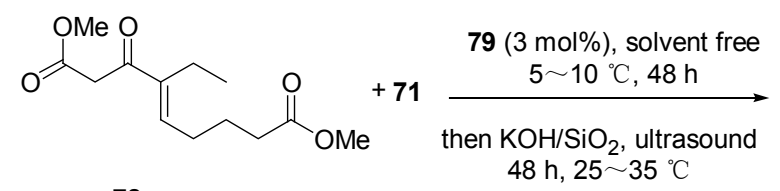

78<smiles></smiles>

反应过程分析发现化合物 80 是先由不对称 Michael 加成的中间体 81 (Scheme 13)转化为酮式环化产物 82, 然后在碱性硅胶作用下中间体 82 发生硝基翻转和酮的 烯醇化转化而来, 其反应机理如 Scheme 14 . 


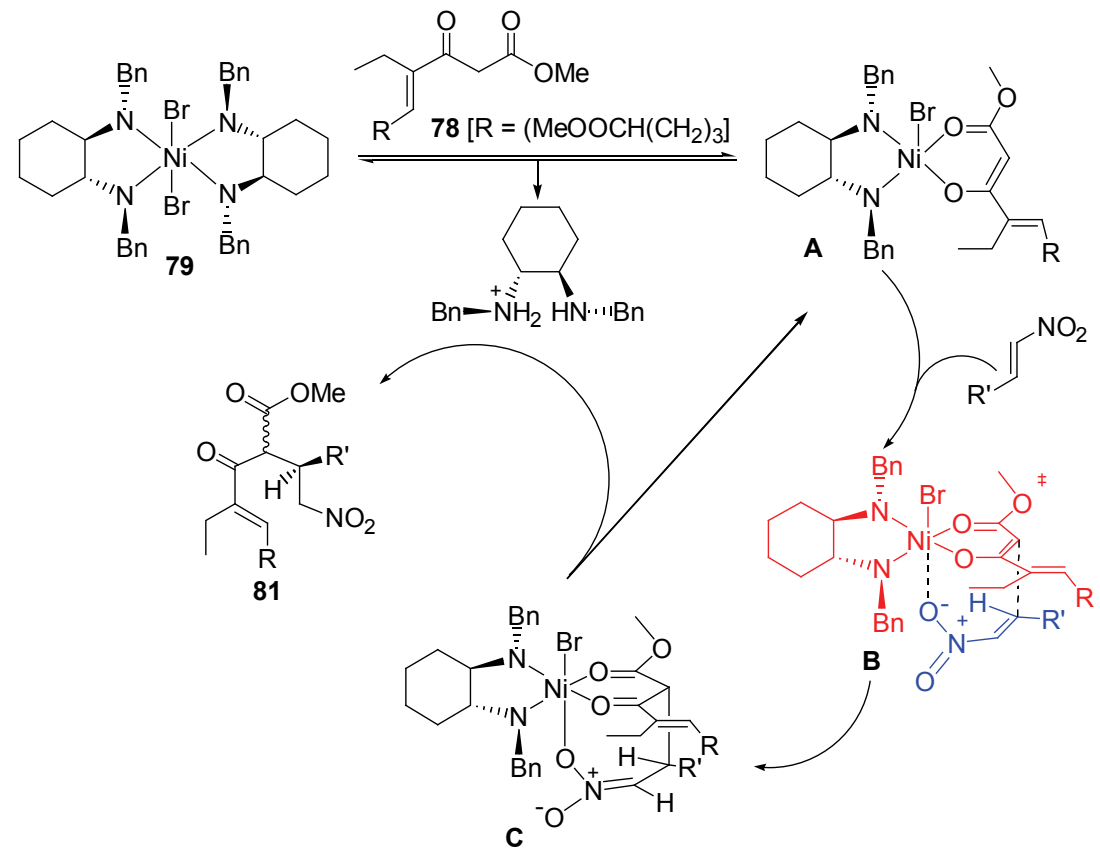

Scheme 13
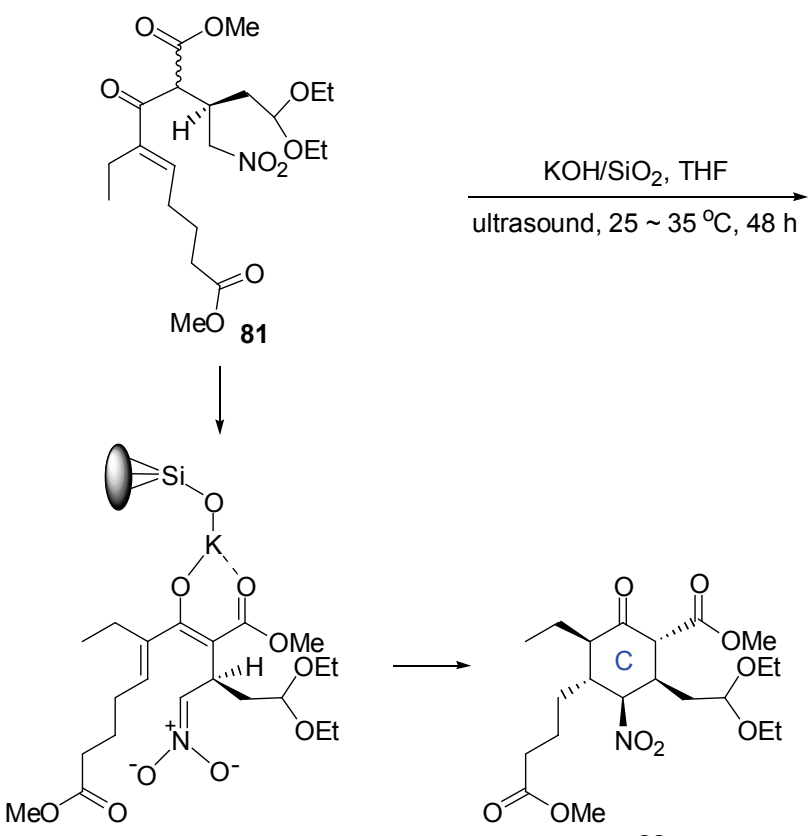<smiles>CCOC(CC1=C(O)C(CC)[C@@H](CCCC(=O)OC)[C@H](CC)C1[N+](=O)[O-])OCC</smiles><smiles>CCCC1C(=O)C(OCC)CC(C(=O)OCC)C1C(=O)OCCCC(=O)OC</smiles><smiles>C=C(OC)C1=C(C)C(CC)[C@H](CCCC(=O)OC)C(=[N+]([O-])[O-])C1CC(CC)OCC</smiles>

Scheme 14

化合物 80 在醋酸(含水)中加热脱去缩醛保护, 然后 加入锌粉, 发生硝基还原和还原胺环化串联反应构建 $\mathrm{AC}$ 二环体系, 粗产物在甲苯中加热经酰胺环化反应生 成具有 $\mathrm{ABC}$ 三环体系结构的化合物 $\mathbf{8 3}$, 此步反应为包 括串联反应在内的共 4 步反应的一瓶反应(One Pot Reaction), 大大提高了合成效率. 化合物 83 经加热脱酯基 得酮化合物 84 , 然后在强碱作用下与溴乙酸乙酯反应 在位阻小的羰基 $\alpha$-位引入乙氧羰基甲基得化合物 85,85 与嗍氢化钠作用, 经羰基还原和内酯化得到具有 $\mathrm{ABCD}$
四环体系结构的化合物 $\mathbf{8 6}$, 化合物 86 在强碱条件下与 碘甲烷作用在酯基的 $\alpha$-位引入甲基生成氧代斯替宁碱 87, 经与 Lawesson 试剂作用转化为硫代斯替宁碱 88, 然后与活性镍作用还原脱硫得到(-)-Stenine (Scheme 15).

到目前为止，此合成路线是唯一利用不对称催化策 略构建斯替宁碱关键骨架结构的方法，串联反应、一瓶 反应等的应用提高了合成效率, 是斯替宁碱高效的不对 称合成方法. 经两次不对称催化 Michael 加成反应生成 


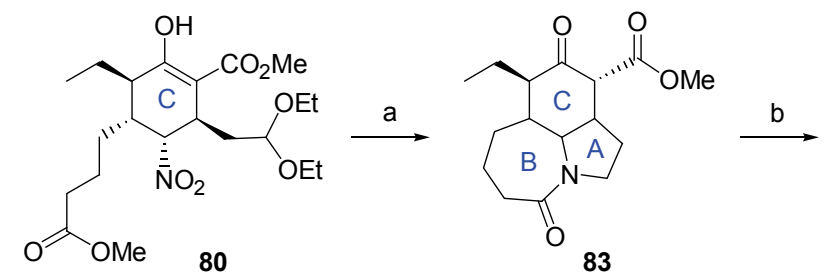<smiles>CCC1C(=O)CC2C(C)CN3C(=O)CCCC(C)C123</smiles>

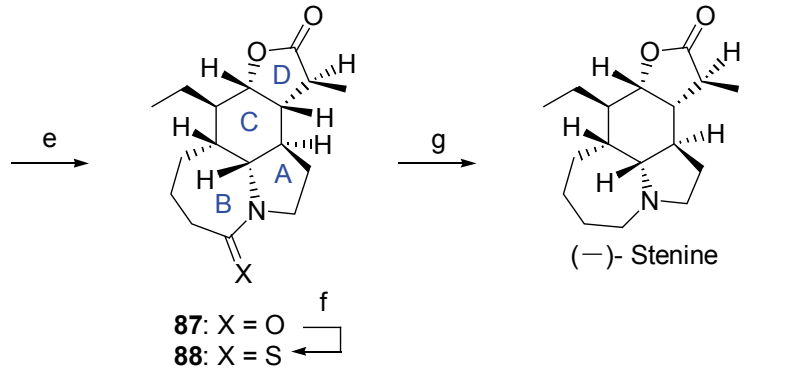

Reagents and conditions: (a) acetic acid, $\mathrm{H}_{2} \mathrm{O}_{2}, 90{ }^{\circ} \mathrm{C}$, then $\mathrm{Zn}$ powder, the crude product was then heated to $90{ }^{\circ} \mathrm{C}$ in toluene (81\%); (b) LiCl, $\mathrm{H}_{2} \mathrm{O}_{2}$, DMSO, $155{ }^{\circ} \mathrm{C}$ (89\%); (c) LiHMDS, THF, $78{ }^{\circ} \mathrm{C}$, ethyl bromoacetate (78\%); (d) $\mathrm{NaBH}_{4}, \mathrm{MeOH}, 0{ }^{\circ} \mathrm{C}(51 \%$ afer recrystallization); (e) LiHMDS, THF, $-78{ }^{\circ} \mathrm{C}, \mathrm{Mel}$ (65\%); (f) Lawesson's reagent, DCM; (g) Raney Ni, EtOH, $25{ }^{\circ} \mathrm{C}(90 \%, 2$ steps)

\section{Scheme 15}

的中间体化合物 82 (控制反应条件, 可以得到较高收率 的此化合物)为五取代环己酮, 具有 5 个连续的手性中 心, 且有很好的对映选择性和非对选择性, 在不对称合 成中具有好的应用前景. 张洪涁研究组从商业化原料出 发, 共计 14 步反应(从已知原料出发, 共 11 步反应), 以 $5.9 \%$ 的总收率合成了 $(-)-$ Stenine.

\section{3 结语与展望}

天然产物、特别是复杂天然产物的全合成是有机化 学最重要的内容之一, 是技术与艺术的综合体现, 是人 类学习自然、超越自然的一个途径, 考验着合成化学家
的能力与智慧. 尽管斯替宁碱的人工全合成从 1990 起, 已经开展了多年的研究，但新的合成方法仍不断地被开 发出来, 正所谓艺无止境, 高效的不对称合成方法仍值 得期待.

复杂天然产物的全合成研究应该遵循变复杂为简 单的原则, 更多地从设计入手, 注重高效、低碳、良好 手性控制等绿色化学特征，开发串联反应、一瓶反应、 手性催化等有机合成方法，为人类利用自然资源，创制 药物等功能分子提供更为可行的合成方法.

\section{References}

[1] For reviews, see: (a) Götz, M.; Strunz, G. M. In Alkaloids, Ed.: Wiesner, K., Butterworth, London, 1973, Vol. 9, pp. 143 160 (b) Lin, W. H.; Ye, Y.; Xu, R. S. J. Nat. Prod. 1992, 55, 571.

(c) Xu, R. S. Nat. Prod. Chem. 2000, 21, 729.

(d) Pilli, R. A.; Ferreira de Oliveira, M. C. Nat. Prod. Rep. 2000, 17, 177.

(e) Greger, H. Planta Med. 2006, 72, 99.

[2] Götz, M.; Edwards, O. E. The Alkaloids, Ed.: Manske, R. H. F., Academic Press, New York, 1967, Vol. 9, pp. 545 550.

[3] (a) Chen, Y. C.; Hart, D. J. J. Org. Chem. 1990, 55, 6236. (b) Chen, Y. C.; Hart, D. J. J. Org. Chem. 1993, 58, 3840.

[4] (a) Wipf, P.; Kim, Y.; Goldstein, D. M. J. Am. Chem. Soc. 1995, $117,11106$.

(b) Goldstein, D. M.; Wipf, P. Tetrahedron Lett. 1996, 37, 739. (c) Wipf, P.; Li, W. J. Org. Chem. 1999, 64, 4576.

[5] Morimoto, Y.; Iwahashi, M.; Nishida, K.; Hayashi, Y.; Shirahama, H. Angew. Chem., Int. Ed. Engl. 1996, 35, 904.

[6] Jung, S. H.; Lee, J. E.; Joo, H. J.; Kim, S. H.; Koh, H. Y. Bull. Korean Chem. Soc. 2000, 21, 159.

[7] (a) Ginn, J. D.; Padwa, A. Org. Lett. 2002, 4, 1515. (b) Ginn, J. D.; Padwa, A. J. Org. Chem. 2005, 70, 5197.

[8] Zeng, Y.; Reddy, D. S.; Hirt, E.; Aubé, J. Org. Lett. 2004, 6, 4993.

[9] (a) Zeng, Y.; Aubé, J. J. Am. Chem. Soc. 2005, 127, 15712. (b) Frankowski, K. J.; Golden, J. E.; Zeng, Y.; Lei, Y.; Aubé, J. J. Am. Chem. Soc. 2008, 130, 6018.

[10] Zhu, L.; Lauchli, R.; Loo, M.; Shea, K. J. Org. Lett. 2007, 9, 2269.

[11] Fujioka, H.; Nakahara, K.; Kotoku, N.; Ohba, Y.; Nagatomi, Y.; Wang, T. L.; Sawama, Y.; Murai, K.; Hirano, K.; Oki, T.; Wakamatsu, S.; Kita, Y. Chem. Eur. J. 2012, 18, 13861.

[12] Chen, J. B.; Chen, J. C.; Xie, Y.; Zhang, H. B. Angew. Chem., Int. Ed. 2012, 51, 1024.

[13] Boeckman, R. K.; Kamenecka, T. M. Jr.; Nelson, S. G.; Pruitt, J. R.; Barta, T. E. Tetrahedron Lett. 1991, 32, 2581. 